\title{
Full-band Monte Carlo simulation of single photon avalanche diodes
}

\author{
Denis Dolgos ${ }^{* 1}$, Hektor Meier ${ }^{2}$, Andreas Schenk ${ }^{3}$, and Bernd Witzigmann ${ }^{4}$ \\ ${ }^{1}$ Synopsys Switzerland LLC Zürich, Switzerland \\ ${ }^{2}$ Albis Optoelectronics AG, Rüschlikon, Switzerland \\ ${ }^{3}$ Integrated Systems Laboratory, ETH Zurich, Switzerland \\ ${ }^{4}$ Computational Electronics and Photonics Group, University of Kassel, Germany
}

Single photon avalanche diodes (SPADs) enable a variety of innovative applications in the fields of biology, medicine, and physics. The propagation of single photons with optical fibers requires the usage of telecommunication wavelengths in the near infrared (NIR) spectral range. NIR SPADs, which can be employed for this application, consist of an absorber layer $\left(\operatorname{In}_{0.53} \mathrm{Ga}_{0.47} \mathrm{As}\right)$ and a multiplication layer ( $\mathrm{InP}$ or $\mathrm{In}_{0.52} \mathrm{Al}_{0.48} \mathrm{As}$ ). The product of the quantum efficiency $\eta_{\mathrm{q}}$, the probability that the photoexcited carrier survives into the multiplier $P_{\mathrm{c}}$, and the breakdown probability $P_{\mathrm{b}}$ that the carrier activates a self-sustaining avalanche designate the photon detection efficiency [1] $\mathrm{PDE}=\eta_{\mathrm{q}} P_{\mathrm{c}} P_{\mathrm{b}}$. The breakdown probability contributes primarily to the electric field dependence of the PDE and increases with the electric field. Therefore, a higher electric field enhances the photon detection efficiency. However, band-to-band or trap-assisted tunneling in the multiplication layer initiate dark counts and degrade the performance of SPAD devices at higher electric fields. Hence, to obtain a higher photon detection efficiency for a given increase of the electric field and tunneling rate, a steep rise of the breakdown probability with applied bias is favorable. The SPAD's timing jitter arises from various sources. The avalanche build-up time is the main contribution to the timing jitter [1].

The full-band Monte Carlo (FBMC) method is considered to be the most accurate device simulation method within the physics of semiclassical charge transport. The FBMC approach for the solution of the Boltzmann transport equation serves as benchmark for approximate methods. FBMC simulations are computationally heavy. This burden forced previous numerical investigations on the SPAD

\footnotetext{
*denis.dolgos@synopsys.com
}

breakdown characteristics to use simplified charge transport models [2-6]. However, nowadays standard computer clusters together with computationally efficient approaches [7] enable the gathering of sufficient statistics with FBMC simulations. The computation of breakdown probabilities and timing jitter has become feasible. The details of our ensemble full-band Monte Carlo simulator CarloS and the approximations of the scattering model have been presented in Refs. [8,9].

We simulate PIN diode structures with intrinsic region widths between $55 \mathrm{~nm}$ and $500 \mathrm{~nm}$ (made of InP, $\mathrm{In}_{0.52} \mathrm{Al}_{0.48} \mathrm{As}$, and GaAs) operated in the Geigermode with a temperature of $T=300 \mathrm{~K}$ [6]. Single carriers are injected with an energy of $10 \mathrm{meV}$ at time $t=0$ ps into the PIN diode. Breakdown occurs when the total number of carriers exceeds 30 within the simulation domain. The simulation stops when a breakdown has not taken place within 500 ps. We have repeated the simulations 2500 times per reverse bias point. Figure 1 presents the breakdown probability versus the excess bias $V_{\mathrm{ex}}=V_{\mathrm{r}}-V_{\mathrm{b}}$ for the different gain materials and multiplication layer widths. We define the breakdown voltage $V_{\mathrm{b}}$ as $P_{\mathrm{b}}\left(V_{\mathrm{b}}\right)=10^{-3}$. Three regions characterize the dependency of the breakdown probability on the reverse bias. First, $P_{\mathrm{b}}$ increases slightly after the breakdown voltage. For higher $V_{\mathrm{r}}$, the breakdown probability depends linearly on the reverse bias. Then, $P_{\mathrm{b}}$ saturates toward unity. The smaller the multiplication region, the steeper the rise of $P_{\mathrm{b}}$ with increasing reverse bias. A steep rise of $P_{\mathrm{b}}$ versus $V_{\mathrm{r}}$ is advantageous because the photon detection efficiency is higher for the same applied excess bias. However, the electric field rises in thinner structures leading to an increased tunneling probability and dark counts. Compared to the simpler models of Refs. [2-6], the FBMC simulations 


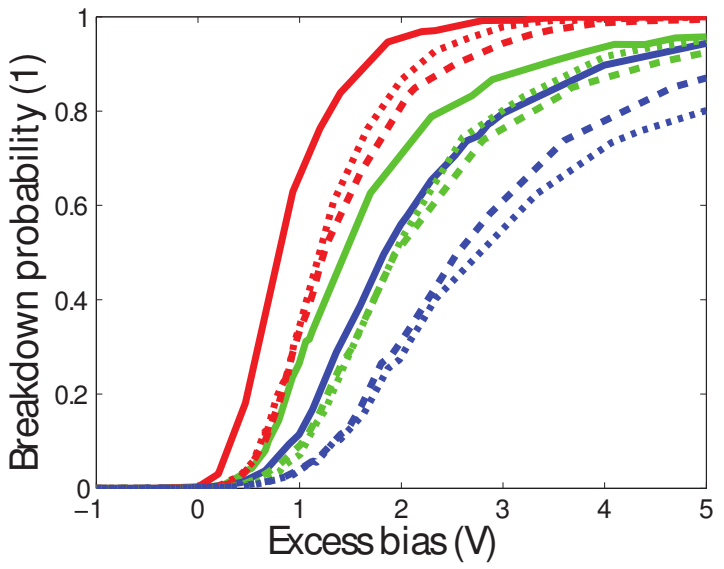

Figure 1: Comparison of the breakdown probability vs. excess bias for $\mathrm{InP}, \mathrm{In}_{0.52} \mathrm{Al}_{0.48} \mathrm{As}$, and GaAs. Red lines: $55 \mathrm{~nm}$, green lines: $250 \mathrm{~nm}$, blue lines: $500 \mathrm{~nm}$, continuous lines: GaAs, dashed lines: $\mathrm{In}_{0.52} \mathrm{Al}_{0.48} \mathrm{As}$, dotted lines: InP.

predict a less steep rise of the breakdown probability with reverse bias. On the one hand, the positive feedback of charge avalanche increases for smaller widths of the gain material and constant excess bias. On the other hand, the effective gain material length is reduced. The balance between these two effects governs the steepness of the breakdown probability for changing multiplication widths. Our FBMC simulations of high-energy charge dynamics of the charge multiplication process reveal the dominance of positive feedback over the reduction of effective gain material width for all three investigated materials [10].

\section{References}

[1] M. Itzler et al., X. Jiang, M. Entwistle, K. Slomkowski, A. Tosi, F. Acerbi, F. Zappa, and S. Cova, "Advances in InGaAsP-based avalanche diode single photon detectors," Journal of Modern Optics, vol. 58, no. 3, pp. 174-200, 2011.

[2] S. Wang, F. Ma, X. Li, G. Karve, X. Zheng, and J. C. Campbell, "Analysis of breakdown probabilities in avalanche photodiodes using a historydependent analytical model," Applied Physics Letters, vol. 82, no. 12, p. 1971, 2003.

[3] D. Ramirez, M. Hayat, G. Karve, J. Campbell, S. Torres, B. Saleh, and M. Teich, "Detection efficiencies and generalized breakdown probabilities for nanosecond-gated near infrared singlephoton avalanche photodiodes," IEEE Journal of Quantum Electronics, vol. 42, no. 2, pp. 137145, 2006.

[4] J. Ng, C. Tan, J. David, and G. Rees, "Theoretical study of breakdown probabilities in single photon avalanche diodes," in IEEE Lasers and Electro-Optics Society (LEOS) Annual Meeting, pp. 773-774, IEEE, 2003.

[5] M. Hayat, U. Sakoglu, J. Campbell, B. Saleh, and M. Teich, "Breakdown probabilities for thin heterostructure avalanche photodiodes," IEEE Journal of Quantum Electronics, vol. 39, no. 1, pp. 179-185, 2003.

[6] S. L. Tan, D. S. Ong, and H. K. Yow, "Theoretical analysis of breakdown probabilities and jitter in single-photon avalanche diodes," Journal of Applied Physics, vol. 102, no. 4, p. 044506 , 2007.

[7] C. Jungemann, S. Keith, M. Bartels, and B. Meinerzhagen, "Efficient full-band Monte Carlo simulation of silicon devices," IEICE Transactions on Electronics, vol. E82C, no. 6, pp. 870-879, 1999.

[8] D. Dolgos, H. Meier, A. Schenk, and B. Witzigmann, "Full-band Monte Carlo simulation of high-energy carrier transport in single photon avalanche diodes: Computation of breakdown probability, time to avalanche breakdown, and jitter," Journal of Applied Physics, vol. 110, no. 8, p. 084507, 2011.

[9] D. Dolgos, A. Schenk, and B. Witzigmann, "Impact ionization scattering model based on the random-k approximation for GaAs, InP, InAlAs, and InGaAs," Journal of Applied Physics, vol. 111 , no. 7 , p. $073714,2012$.

[10] D. Dolgos, H. Meier, A. Schenk, and B. Witzigmann, "Full-band Monte Carlo simulation of high-energy carrier transport in single photon avalanche diodes with multiplication layers made of InP, InAlAs, and GaAs," Journal of Applied Physics, vol. 111, no. 10, p. 104508, 2012. 